\title{
SPHERICAL BUNDLES ADAPTED TO A $G$-FIBRATION
}

\author{
BY
}

J. P. E. HODGSON

\begin{abstract}
A spherical fibration $p: E \rightarrow B$ is said to be adapted to a $G$-fibration $\pi$ : $E \rightarrow E / G$ if there is a fibration $q: E / G \rightarrow B$ with fibre the quotient of a sphere by a free $G$-action and such that the composition $q \circ \pi=p$. In this paper it is shown that for spherical bundles in the PL, TOP or Homotopy categories that are adapted to $Z_{2}$ - or $S^{1}$-fibrations there is a procedure analogous to the splitting principle for vector bundles that enables one to define characteristic classes for these fibrations and to relate them to the usual characteristic classes. The methods are applied to show that a spherical fibration over a 4-connected base which is adapted to an $S^{1}$-fibration admits a PL structure.
\end{abstract}

Introduction. In the classical case of a real or complex vector bundle, the associated spherical bundles admit a free fibrewise action by the elements of unit norm in the ground field. The quotient space of this action is the projectivisation of the original vector bundle. It is well known that by pulling back the original bundle to a bundle over the projectivisation, one can split off a line bundle. This procedure is the genesis of the splitting principle for vector bundles, and it can be exploited to define characteristic classes for the original bundle. This paper examines the situation in other categories of spherical bundles. The immediate problem, of course, is that in these cases we do not, in general, have the free fibrewise group action of the vector bundle case. To remedy this, we introduce the notion of a spherical bundle adapted to a $G$-fibration; in essence this is just a spherical bundle $p: E \rightarrow B$ which is also the total space of a $G$-fibration $\pi: E \rightarrow E / G$, such that $E / G$ is a bundle over $B$ whose fibre is the quotient of a sphere by a free $G$-action. The notion makes sense in the Piecewise Linear, Topological and Homotopy categories. We are able to describe a quasi-splitting principle for these bundles, that mimics the splitting principle; even though the pull-back over the "projectivisation" $E / G$ does not split! We can thus define characteristic classes for spherical bundles adapted to $G$-fibrations and we relate them to the usual characteristic classes for spherical bundles. Finally, we are able to apply our methods to show that a spherical fibration over a 4-connected base that admits a free fibrewise $S^{1}$ action lifts to a PL bundle. This is a special case of a conjecture of Sullivan.

The paper is arranged as follows: $\$ 1$ consists of definitions and notations, and introduces the notion of a spherical bundle adapted to a $G$-fibration. $\$ 2$ describes

Received by the editors October 24, 1979.

1980 Mathematics Subject Classification. Primary 55R25, 57S17; Secondary 57Q65.

Key words and phrases. Block bundle, spherical fibration, projectivisation, characteristic classes, transversality obstructions. 
the quasi-splitting principle and in $\$ 3$ we apply the principle to construct characteristic classes; in particular, we show that for $G=S^{1}$ the construction yields the Pontrjagin classes of Topological and PL bundles. $\$ 4$ is devoted to the proof of Sullivan's conjecture.

1. Definitions and notations. Let $\mathcal{C}$ denote one of the following three categories:

PL: the category of polyhedra and polyhedral maps.

Top: the category of topological spaces and continuous maps.

$\mathrm{Htp}$ : the category of $\mathrm{CW}$-complexes and homotopy classes of maps.

A $C$-block bundle with fibre $F$ over the polyhedron $B$ is an equivalence class of projections $p: E \rightarrow B$ such that there is a triangulation of $B$ with a $C$-isomorphism $\left\{p^{-1}(\sigma), p^{-1}(\partial \sigma)\right\}=F \times(\sigma, \partial \sigma)$, for each simplex in the triangulation. The equivalence relation on the projection is defined in [1] for the case $\mathcal{C}=\mathrm{PL}$; it is not difficult to see how to adapt the relation to the other categories.

The spaces $p^{-1}(\sigma)$ are called the blocks of $E$. It is convenient to regard $E$ as being built up by gluing together the blocks $\sigma \times F$ across the faces $\partial \sigma \times F$ using equivalences from the category $C$.

The model for our next definition is a spherical fibration on which a compact Lie group $G$ acts freely and in a fibrewise manner. In the case of the associated sphere bundle of a vector bundle with a smooth $G$-action, the quotient space is a bundle whose fibre is $S^{k} / G$. It is this observation that is embodied in the definition.

Definitíon 1.1. Let $p: E \rightarrow B$ be a $\mathcal{C}$-block bundle with fibre $S^{k}$, and $G$ a compact Lie group. Then $p: E \rightarrow B$ is a spherical bundle adapted to a $G$-fibration, if there exist a $G$-fibration $\pi: E \rightarrow E / G$ and a map $p / G: E / G \rightarrow B$ which is a C-block bundle with fibre $S^{k} / G$ such that the following diagram commutes.

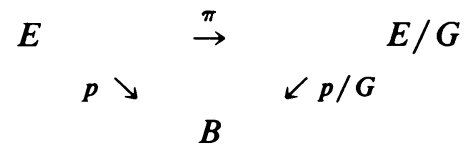

For a fixed group $G$, spherical fibrations adapted to a $G$-fibration form a reasonable category, in particular they admit pull-backs.

Definition 1.2. If $p: E \rightarrow B$ is a spherical bundle adapted to a $G$-fibration, and $G=Z_{2}$ or $S^{1}$, we shall call the bundle $p / G: E / G \rightarrow B$ the projectivisation of $E$, and we shall write $P(E)$ for $E / G$. This corresponds to the classical terminology for vector bundles.

We need a special notion of subbundle for the category of spherical bundles adapted to $G$-fibrations; this is given by the following definition.

Definition 1.3. Let $p: E \rightarrow B$ be a spherical bundle adapted to a $G$-fibration so that we have a commutative diagram

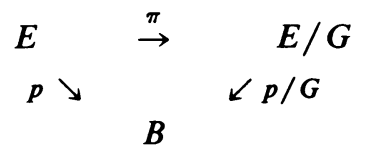

then a subbundle $p_{0}: E_{0} \hookrightarrow E \rightarrow B$ is an adapted subbundle of $p: E \rightarrow B$ if $p_{0}$ is itself adapted to a $G$-fibration $\pi_{0}: E_{0} \rightarrow E_{0} / G$ and there is a commutative diagram 


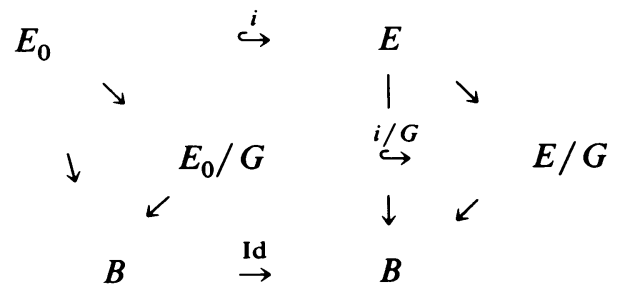

We have the following proposition:

Proposition 1.4. Let $G$ be one of the groups $Z_{2}$ or $S^{1}$, and let $d=1$ (resp. $d=2$ ) if $G=Z_{2}$ (resp. $G=S^{1}$ ). Suppose that $p: E \rightarrow B$ is a spherical bundle adapted to $a$ $G$-fibration with projectivisation $p / G: P(E) \rightarrow B$. Then the induced spherical bundle $(P / G)^{*}(E)$ over $P(E)$ is adapted to a $G$-fibration and has an adapted subbundle of rank $d$. (This subbundle is, in fact, a $G$-fibration over $P(E)$.)

Proof. Consider the pull-back diagram.

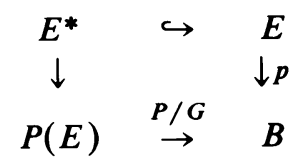

Then $E^{*}=\{(x, y) \in E \times P(E) \mid p(x)=(p / G)(y)\}$ is the total space of $(p / G)^{*} E$. If $\pi: E \rightarrow P(E)$ is the projection in the $G$-fibration, then $T=$ $\{(x, \pi(x))\} \subset E^{*}$ with the projection $q: T \rightarrow P(E)$ given by $q(x, \pi(x))=\pi(x)$ is a copy of $E$ in $E^{*}$ which is the required adapted subbundle of rank $d$.

In the classical case where $E$ is the associated sphere bundle of a vector bundle and $G$ acts linearly, then the subbundle given by the above proposition splits off. Repetition of this process gives the well-known splitting principle [2]. Unfortunately, in the categories $\mathcal{C}$, with which we are concerned, we cannot suppose that this subbundle splits off. The next section describes a way of circumventing this difficulty so as to obtain a process parallel to the splitting principle.

2. A quasi-splitting principle. In this and all subsequent sections we restrict ourselves to the cases $G=Z_{2}$ and $G=S^{1}$. It will be convenient to define $d=1$ if $G=Z_{2}$, and $d=2$ if $G=S^{1}$; also $F=\mathbf{R}$ if $G=Z_{2}$ and $F=\mathbf{C}$ if $G=S^{1}$.

Proposition 1.4 leads us to consider the following situation. We suppose given a spherical bundle $p: E \rightarrow B$ adapted to a $G$-fibration $\pi: E \rightarrow P(E)$. Further, we suppose that $p: E \rightarrow B$ contains a (locally flat) adapted subbundle of rank $d, l$ : $L \rightarrow B$. Let $P(L)$ denote the projectivisation of $L$.

In the cases $C=$ PL or Top, $P(L)$ has a block bundle neighbourhood $\nu(P(L))$ in $P(E)$. We need a comparable result in the remaining case $C=H$ tp. This is given by the following lemma.

Lemma 2.1. Given a spherical bundle $p: E \rightarrow B$ adapted to a G-fibration in Htp, which contains an adapted subbundle $l: L \rightarrow B$ of rank $d$, we can find a spherical bundle $p^{\prime}: E^{\prime} \rightarrow B$ adapted to a $G$-fibration and containing an adapted subbundle $l^{\prime}$ : $L^{\prime} \rightarrow B$ such that the adapted bundle pair $\left(E^{\prime}, L^{\prime}\right)$ is equivalent to $(E, L)$. Furthermore, $P\left(L^{\prime}\right)$ has a block bundle neighbourhood in $E^{\prime}$, and boundary of this neighbourhood is a spherical fibration over $L^{\prime}$. 
Proof. The proof is by induction over the cells of $B$. Triangulate $B$ and order the simplexes in increasing order of dimension. This gives a corresponding inductive construction for $P(E)$ obtained by gluing copies of $\sigma_{k} \times P_{n-1}(F)\left(\sigma_{k}=\right.$ a $k$-simplex, and $P_{n-1}(F)$ is the $(n-1)$-dimensional projective space over $\left.F\right)$ to the already constructed portion. Furthermore, we know that in this construction the subblocks $\sigma_{k} \times P_{0}(F)$ corresponding to $P(L)$ are also glued together; thus, the attaching maps that build $P(E)$ are maps of the form

$$
\alpha: \partial \sigma_{k} \times\left(P_{n-1}(F), P_{0}(F)\right) \rightarrow \partial \sigma_{k} \times\left(P_{n-1}(F), P_{0}(F)\right) .
$$

We shall show that $\alpha$ can be homotoped so that the (normal space) transverse inverse image under $\alpha$ of $\partial \sigma_{k} \times P_{0}(F)$ is $\partial \sigma_{k} \times P_{0}(F)$. This will give the lemma because it shows that $\alpha$ respects the normal bundle of $\partial \sigma_{k} \times P_{0}(F)$ in $\partial \sigma_{k} \times$ $P_{n-1}(F)$.

To see our claim about the normal space transverse inverse image under $\alpha$ of $\partial \sigma_{k} \times P_{0}(F)$, we note that for this case of equal dimensions Quinn's definition [8] of normal space transversality gives the inverse image $\alpha^{-1}\left(\partial \sigma_{k} \times P_{0}(F)\right)$ a normal space structure. Normal space surgery on $\alpha^{-1}\left(\partial \sigma_{k} \times P_{0}(F)\right)$ is performed by taking a sequence of cofibrations. Now $\alpha^{-1}\left(\partial \sigma_{k} \times P_{1}(F)\right)$ contains $\partial \sigma_{k} \times P_{0}(F)$ so that there is a cofibration $\partial \sigma_{k} \times P_{0}(F) \rightarrow \alpha^{-1}\left(\partial \sigma_{k} \times P_{0}(F)\right)$. Furthermore, this cofibration preserves fundamental classes, so that it can be achieved by normal space surgery.

In this way we can construct a normal bundle for $P(L)$ in $P(E)$ as required.

Proposition 2.2. The block bundle $\eta: \partial \nu(P(L)) \rightarrow B$ obtained by taking the boundary of the normal bundle to $P(L)$ in $P(E)$ has the structure of a spherical bundle adapted to a $G$-fibration.

Proof. $P(E)$ is a block bundle whose blocks are of the form $\sigma \times P_{n-1}(F)$ for some $n$. Each block contains a subblock $\sigma \times P_{0}(F)$ which is the corresponding block of $P(L)$. Thus from Lemma 2.1 $P(E)$ - Int $\nu(P(L))$ is expressed as a union of blocks $\sigma \times\left(P_{n-1}(F)-\operatorname{Int} D^{d(n-1)}, \partial\left(P_{n-1}(F)-\right.\right.$ Int $\left.\left.D^{d(n-1)}\right)\right)$ and these have spines $\sigma \times P_{n-2}(F)$. Proceeding by induction over the interiors of simplexes of $B$ in descending order of dimension we can, thus, construct a block bundle $\mu: K \rightarrow B$ with fibre $P_{n-2}(F)$ by gluing these spines together. This gives us a block fibration $\lambda: \partial \nu(P(L)) \rightarrow K$ which is a $G$-block bundle, where $\lambda$ is the composition of the inclusion $\partial \nu(P(L)) \rightarrow P(E)-\operatorname{Int} \nu(P(L))$ with the map collapsing $P(E)-$ Int $\nu(P(L))$ to $K$. This realises $\eta$ as a spherical bundle adapted to a $G$-fibration.

Denote by $T(l * \eta)$ the Thom space of the spherical bundle $l * \eta$ which is the Whitney join of the rank $d$ bundle $l: L \rightarrow B$ and the bundle $\eta$ of Proposition 2.2. $T(p)$ will denote the Thom space of the original bundle $p: E \rightarrow B$. Also, let $B(l * \eta)$ (resp. $B(p)$ ) denote the disc bundles associated to $l * \eta$ (resp. $p: E \rightarrow B$ ).

Proposition 2.3. There is a map $\alpha: B(l * \eta) \rightarrow B(p)$ whose restriction to $B$ is homotopic to the identity and inducing a map $\tilde{\alpha}: T(l * \eta) \rightarrow T(p)$ which preserves Thom classes. 
Proof. It suffices to construct a map of the total space of the spherical fibration $l * \eta$ into $B(p)-B$ that extends the inclusion of $L$ into $E$. That this can be done follows by straightforward homotopy theory, upon observing that a copy of the pull-back $l^{*}(\eta)$ lies in $E$ as the boundary of a neighbourhood of $L$, and that $E-\nu(L)$ has the homotopy type of the total space of $\eta$.

COROLlaRY. In the above notation $W(l * \eta)=W(p)$ where $W$ denotes the total Stiefel-Whitney class.

3. Characteristic classes. Given a spherical bundle $p: E \rightarrow B$ adapted to a $G$-fibration $\pi: E \rightarrow E / G$, then using the quasi-splitting principle of $\$ 2$ we can associate to $p: E \rightarrow B$ a join of rank $d$ bundles. This is done as follows: $p / G: E / G$ $=P(E) \rightarrow B$ is the projectivisation of $p: E \rightarrow B$ and so by Proposition 1.4 the induced bundle $(p / G)^{*}(E)$ over $P(E)$ satisfies the hypotheses of Lemma 2.1, and we have the map $\alpha: B(l * \eta) \rightarrow B(p)$ of the associated disc bundles. Furthermore, $\eta$ itself is a spherical bundle adapted to a $G$-fibration by Proposition 2.2, so we can repeat the argument. Iteration thus gives us a bundle $*_{i=1}^{k} l_{i}$ over a space $F(E)$, where each $l_{i}$ is a spherical bundle of rank $d$, and $F(E)$ is obtained by a succession of block bundles whose fibres are projective spaces. This fibration $\mu: F(E) \rightarrow B$ is called the quasi-flag bundle of $p: E \rightarrow B$. Furthermore, Proposition 2.3 gives us a map $\alpha: B\left(*_{i=1}^{k} l_{i}\right) \rightarrow B\left(\mu^{*}(p)\right)$ between the disc bundles associated to $*_{i=1}^{k} l_{i}$ and the disc bundle associated to the bundle $\mu^{*}(p)$ induced by $\mu$ from $p: E \rightarrow B$. This map $\alpha$ is called a quasi-splitting of $p: E \rightarrow B$.

Proposition 3.1. The map $\mu: F(E) \rightarrow B$ induces a monomorphism in cohomology for $Z_{2}$ coefficients in case $G=Z_{2}$ and for $Z$-coefficients in case $G=S^{1}$.

Proof. This is the Thom-Dold theorem [3].

The following are the main results of this section.

TheOREM 3.2. Let $p: E \rightarrow B$ be a spherical fibration adapted to an $S^{1}$ fibration, in the categories Top or PL; and let $\alpha: B\left(*_{i=1}^{k} l_{i}\right) \rightarrow B\left(\mu^{*}(p)\right)$ be the associated quasi-splitting. Then the rational Pontrjagin classes of $\mu^{*}(p)$ and $*_{i=1}^{k} l_{i}$ in $H_{*}(F(E) ; \mathbf{Q})$ are equal.

TheOREM 3.3. If $p: E \rightarrow B$ is a spherical bundle adapted to a $G$-fibration, then the quasi-splitting principle can be used to define rational Pontrjagin classes.

Theorem 3.3 follows easily from Theorem 3.2, Proposition 3.1, and the corollary to Proposition 2.3. In the case $e=H$ tp, we observe that since rank 2 spherical fibrations are $S^{1}$ fibrations, they have Chern classes and, thus, we can define Pontrjagin classes for $*_{i=1}^{k} l_{i}$ in this case as well.

Proof of Theorem 3.2. We consider first the piecewise linear case. It suffices to show that in the situation of $\$ 2$ the rational Pontrjagin classes of $\eta * l$ equal the rational Pontrjagin classes of $p: E \rightarrow B$, as bundles over $B$. According to Sullivan [9], the rational Pontrjagin classes are determined by the diagram 


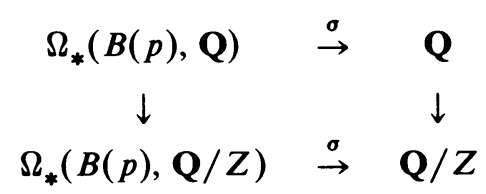

where the map $\sigma$ is determined by taking the index of the intersection with the zero-section. Now we have the map $\alpha: B(\eta * l) \rightarrow B(p)$ which by construction induces an isomorphism $\alpha_{*}: \Omega_{*}(B(\eta * l)) \rightarrow \Omega_{*}(B(p))$. So it suffices to observe that since $\alpha$ maps $\eta * l$ into $B(p)-B$ the diagram

$$
\begin{array}{ccc}
\Omega_{*}(B(\eta * l), \mathbf{Q}) & \stackrel{\alpha_{*}}{\rightarrow} & \Omega_{*}(B(p), \mathbf{Q}) \\
\boldsymbol{\sigma}_{\eta, l} \searrow & & \swarrow \sigma_{p}
\end{array}
$$

Q

commutes, as does the corresponding diagram for $\mathbf{Q} / Z$. In the case that $p: E \rightarrow B$ is a topological bundle, the above argument is obstructed by the failure of transversality in dimension (or codimension) four in the topological category. These difficulties can be avoided by multiplying the base by $\mathbf{C} P^{2}$ (for the case where the base is four dimensional) and by adding a trivial bundle (for the case where the rank is four). Neither of these operations changes the Pontrjagin classes, so we can apply the foregoing argument to obtain the result.

4. A conjecture of Sullivan. This paragraph is devoted to a discussion of the following conjecture, which is due to D. Sullivan.

Conjecture. Suppose $p: E \rightarrow B$ is a spherical fibration upon which $S^{1}$ acts freely in a fibrewise manner; then $p: E \rightarrow B$ admits a PL block bundle structure.

The main result of this paragraph is the following theorem.

TheOREM 4.1. Let $p: E \rightarrow B$ be a spherical bundle in Htp, which is adapted to an $S^{1}$ fibration, and suppose that $B$ is 4-connected; then $p: E \rightarrow B$ admits a PL block bundle structure.

This implies that the Sullivan conjecture is true over a 4-connected base. Before giving the details, we describe the idea of the proof.

IDEA OF PROOF. We begin by applying the quasi-splitting principle to the bundle $p: E \rightarrow B$; this gives us a map $\alpha: B\left(*^{k}{ }_{i=1} l_{i}\right) \rightarrow B\left(\mu^{*}(p)\right)$ where $\mu: F(E) \rightarrow B$ is the quasi-flag bundle associated to $p: E \rightarrow B$. Now $\alpha$ enables us to define Pontrjagin classes for $\mu^{*}(p)$; in fact, we shall show that one can go further and produce a transversal structure in the sense of Levitt-Morgan [5] on $\mu^{*}(p)$. The results of Levitt-Morgan and the fact that $B$ is 4-connected then show that $\mu^{*}(p)$ lifts to a PL bundle. The proof is completed by showing that $\mu^{*}:[B ; B(F /$ Top $)] \rightarrow$ $[F(E) ; B(F /$ Top $)]$ is a monomorphism so that $p: E \rightarrow B$ also lifts to a PL bundle. (Recall that the base is assumed 4-connected, so lifting to PL and Top is the same.)

Proof of Theorem 4.1. As described in the outline above, we have the map

$$
\alpha: B\left(\begin{array}{cc}
k & \\
* & l \\
i=1
\end{array}\right) \rightarrow B\left(\mu^{*}(p)\right)
$$

where each $l_{i}$ is a spherical bundle of rank 2. Each $l_{i}$ has a transversal structure 
since $G_{2} / \mathrm{PL}_{2} \simeq *$. The map $\alpha$ gives rise to a map $\tilde{\alpha}: T\left(*_{i=1}^{k} l_{i}\right) \rightarrow T\left(\mu^{*}(p)\right)$ of the associated Thom spaces. Suppose that we have a map $f: \Delta^{l} \rightarrow T\left(\mu^{*}(p)\right)$ which is transverse to the zero-section when restricted to $\partial \Delta^{l}$. Recall that the Levitt theory of obstruction to transversality [4] evaluates the obstruction cocycle on $f$ by comparing $\left(f / \partial \Delta^{l}\right)^{-1}$ (zero-section) with the inverse image obtained by using $f$ as a null-homotopy of $f / \partial \Delta^{l}$ to make $f$ PL transverse on all of $\Delta^{l}$. We claim that there is a map $g: \Delta^{l} \rightarrow T\left(*_{i=1}^{k} l_{i}\right)$ such that the following diagram commutes.

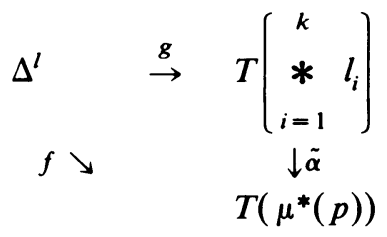

This is because $\alpha$ is a homotopy equivalence. In fact, since $\alpha$ was constructed so that $*_{i=1}^{k} l_{i}$ maps into $B\left(\mu^{*}(p)\right)-F(E)$, we could have constructed $\alpha$ so as to map $*_{i=1}^{k} l_{i}$ into the complement of a neighbourhood of $F(E)$, thus, giving a specific homotopy inverse $\tilde{\gamma}: T\left(\mu^{*}(p)\right) \rightarrow T\left(*_{i=1}^{k} l_{i}\right)$ and $g$ can be taken as $\tilde{\gamma} \circ f$.

Now if we consider $\left(f / \partial \Delta^{l}\right)^{-1}(F(E))$ this is equal to $\left(g / \partial \Delta^{l}\right)^{-1}(F(E))$ by the construction of $\tilde{\alpha}$ and $g$ is just a shift of $f$ to a PL transversal map. But this shift has been achieved without changing $\left(f / \partial \Delta^{l}\right)^{-1}(F(E))$. Hence, the Levitt obstruction to transversality vanishes, and $\mu^{*}(p)$ has a transversal structure. By the results of Levitt-Morgan [5] $\mu^{*}(p)$ lifts to BPL. The theorem will be proved if we show the following lemma.

LeMMA 4.2. Let $p: E \rightarrow B$ be a spherical fibration adapted to an $S^{1}$-fibration, and let $\mu: F(E) \rightarrow B$ be the associated quasi-flag bundle; then the induced map $\mu^{*}$ : $[B, B(G /$ Top $)] \rightarrow[F(E), B(G /$ Top $)]$ is mono.

Proof. This follows from the Thom-Dold theorem [3], and the two observations that at odd primes $B(G /$ Top) is $\mathrm{BBO}[10]$ and at even primes by results of Madsen and Milgram [6] $B(G /$ Top) is a product of Eilenberg-Mac Lane spaces.

\section{REFERENCES}

1. E. Akin, Transverse cellular maps of polyhedra, Trans. Amer. Math. Soc. 169 (1972), 401-438.

2. M. F. Atiyah, K-theory, Benjamin, New York, 1967.

3. A. Dold, Relations between ordinary and extraordinary cohomology, Proc. Aarhus Sympos., Aarhus Univ., Aarhus, 1962, pp. 2-9.

4. N. Levitt, Poincaré duality cobordism, Ann. of Math. (2) 96 (1972), 211-244.

5. N. Levitt and J. Morgan, Transversality structures and PL structures on spherical fibration, Bull. Amer. Math. Soc. 78 (1972), 1064-1068.

6. I. Madsen and R. J. Milgram, The universal smooth surgery class, Comment. Math. Helv. 50 (1975), 281-310.

7. J. Milnor and J. Stasheff, Characteristic classes, Ann. of Math. Studies, no. 76, Princeton Univ. Press, Princeton, N. J., 1974.

8. F. Quinn, Surgery on Poincaré and normal spaces, Bull. Amer. Math. Soc. 78 (1972), 262-267.

9. D. Sullivan, Geometric homotopy theory. I. Localization, Periodicity and Galois Symmetry, M.I.T. Press, Cambridge, Mass., 1970.

10. __ Geometric topology, Lecture Notes, Princeton Univ., Princeton, N. J., 1967.

Department of Mathematics, Adelphi University, Garden City, New York 11530 